\title{
Environmental factors affecting toxic shock syndrome toxin-1 (TSST-1) synthesis
}

\author{
S. K. SARAFIAN* and S. A. MORSE
}

\begin{abstract}
Department of Microbiology and Immunology, Emory University, Atlanta and Sexually Transmitted Diseases Laboratory Program Center for Infectious Diseases, Centers for Disease Control, Atlanta, GA 30333, USA
\end{abstract}

\begin{abstract}
Summary. The production of toxic shock syndrome toxin-1 (TSST-1) was studied in batch and continuous culture of Staphylococcus aureus strain 1169 in a carbohydratefree chemically defined medium (CDM). In continuous culture oxygen- and argininelimitation were required for steady-state TSST-1 synthesis. Aeration suppressed toxin synthesis. The amount of TSST-1 per mg dry weight (specific toxin) at dilution rates from 0.05 to $0 \cdot 15 \mathrm{~h}^{-1}$ was inversely proportional to the dilution rate. Protease activity increased with increasing dilution rates. In batch culture, TSST-1 began to accumulate in the medium towards the end of the exponential phase of growth, after a critical cell mass was attained. Maximum specific toxin production was observed in medium with an initial $p \mathrm{H}$ between 6.5 and $7 \cdot 0$. Growth and toxin synthesis took place in anaerobic conditions when CDM was supplemented with pyruvate and uracil. The $\mathrm{Mg}^{++}$concentration had no effect on the specific toxin in anaerobic conditions. In aerobic conditions, specific toxin increased c. 23 -fold as the $\mathrm{Mg}^{++}$concentrations increased to $0.4 \mathrm{mM}$. Further increases in the $\mathrm{Mg}^{++}$concentration resulted in a reduction in specific toxin.
\end{abstract}

\section{Introduction}

Toxic shock syndrome (TSS) was first described by Todd et al. (1978) who defined criteria to distinguish this multisystem illness from other similar diseases. Although the pathogenesis of TSS remains unclear, it is often associated with strains of Staphylococcus aureus capable of producing toxic shock syndrome toxin-1 (TSST-1), and an illness very similar to TSS has been described in some animal models (Scott et al., 1983; Arko et al., 1984; Reeves et al., 1984; Crass and Bergdoll, 1986). Factors affecting TSST-1 synthesis have been the subject of few studies and are still largely unknown (Schlievert and Blomster, 1983; Mills et al., 1985; Schlievert, 1985). All previous investigations have used staphylococci grown in batch culture which has several disadvantages. Batch cultures are closed systems in which the environment is constantly changing because of the depletion of nutrients and accumulation of end products. Furthermore, batch cultures are time-dependent; the cells and their products from the exponential phase of growth may

Received 3 Sep. 1986; accepted 18 Sep. 1986.

* Correspondence should be sent to Dr S. K. Sarafian, Building 1, Room 3329, Centers for Disease Control, Atlanta, GA 30333, USA. not be the same as those from the stationary phase of growth. These problems are avoided in continuous culture where the growth rate is controlled by the rate at which a growth-limiting nutrient is added to the culture.

TSST-1 synthesis has hitherto been examined with staphylococci grown in nutrient excess and, consequently, with a rapid generation time. In comparison, the growth rate of micro-organisms in mammalian tissues has been shown to be very slow (Maw and Meynell, 1968; Polk and Miles, 1973) which suggests that, in vivo, the growth rate is limited by the availability of substrates. This study was designed to determine the effect of a controlled environment on TSST-1 synthesis. The effects of anaerobiosis, pyruvate, and magnesium were also examined in batch culture.

\section{Materials and methods}

\section{Bacteria}

$S$. aureus strains 1169 and 033 were obtained from M. S. Bergdoll (Food Research Institute, University of Wisconsin, WI, USA) and R. J. Arko (Sexually Transmitted Diseases Laboratory Program, Centers for Disease Control, Atlanta, GA, USA) respectively. With chemically defined medium (Catlin, 1973), we found that strain 1169 required arginine and proline for growth. Bacteroides 
fragilis strain 860009 was provided by V. R. Dowell (Centers for Disease Control, Atlanta, GA).

\section{Medium and growth conditions}

The chemically defined medium (CDM) described by Reeves et al. (1984) was used in all experiments. The medium was sterilised by filtration (prefilter and $0 \cdot 22-\mu \mathrm{m}$ pore size filter; Millipore, Bedford, MA, USA). Aerobic batch cultures were grown in 250-ml Klett flasks, each containing $50 \mathrm{ml}$ of CDM. The flasks were incubated at $37^{\circ} \mathrm{C}$ in a rotary shaking water bath at $150 \mathrm{rpm}$.

Anaerobic cultures were grown in 50-ml tubes fitted with loose metal caps; each tube contained $10 \mathrm{ml}$ of CDM. After inoculation, the medium was flushed for 1 min with $\mathrm{N}_{2} 95 \%, \mathrm{CO}_{2} 5 \%$ which had been sterilised by filtration. The tubes were then placed in an anaerobic jar with a gas-generating sachet (Gas Pak; BBL Microbiology Systems, Cockeysville, MD, USA) and incubated at $37^{\circ} \mathrm{C}$ on a rotary shaker at $150 \mathrm{rpm}$. B. fragilis was included in these experiments as a control for anaerobic conditions.

\section{Continuous culture}

Initial experiments indicated that growth depended on the availability of amino acids. Thus, the CDM used for continuous culture was modified to contain one eighth the amount of amino acids present in the original medium. The concentration of arginine was such as to be the growth-rate-limiting nutrient. Staphylococci were grown in model C-30 and C-32 chemostats (New Brunswick Scientific Co., New Brunswick, NJ, USA) equipped with $350-\mathrm{ml}$ or $1350-\mathrm{ml}$ culture vessels, respectively. Dissolved oxygen (DO) was monitored with a 900 series dissolved oxygen electrode (New Brunswick Co.). Before inoculation, the medium was saturated with oxygen by adjusting the air flow to $400 \mathrm{ml} / \mathrm{min}$ and the impeller speed to $800 \mathrm{rpm}$. Inocula consisted of a suspension of $S$. aureus strain 1169 dispensed in 1-ml volumes and stored at $-70^{\circ} \mathrm{C}$. After thawing, the suspension was used to inoculate the chemostat. The culture was kept at $37^{\circ} \mathrm{C}$ for $10 \mathrm{~h}$ with an air flow of $400 \mathrm{ml} / \mathrm{min}$ and an impeller speed of $400 \mathrm{rpm}$. After $10 \mathrm{~h}$, the nutrient pump was started and the impeller speed increased to $700-800 \mathrm{rpm}$. Foaming was controlled by the addition of Antifoam $\mathrm{C}$ emulsion (Sigma Chemical Co., St Louis, MO, USA) to the growth medium before filter sterilisation. The final dilution of the antifoam $\mathrm{C}$ in the medium was 1 in $10^{6}$. To ensure the attainment of a steady state, staphylococcal cultures were allowed to remain at each dilution rate until at least 2 volumes of medium had passed through the culture vessel.

\section{Toxin assay}

Culture supernates were obtained after centrifugation at $5000 \mathrm{~g}$ for $10 \mathrm{~min}$ at $4^{\circ} \mathrm{C}$. The culture supernates were concentrated 25 - or 50 -fold by ethanol precipitation
(Reeves et al., 1984). The concentration of TSST-1 was determined by a radial immunodiffusion assay with goat antitoxin diluted 1 in 30 (Mancini et al., 1965). The gel diffusion plates were incubated at room temperature and read after $24 \mathrm{~h}$. The goat antitoxin was provided by M. W. Reeves (Centers for Disease Control). Reference toxin standards containing $12 \cdot 5,25$, and $50 \mu \mathrm{g}$ of TSST$1 / \mathrm{ml}$ were prepared by diluting purified TSST- 1 in phosphate-buffered saline, $p \mathrm{H}$ 7-2. A reference curve was plotted for each experiment. The purified TSST-1 was obtained from J. Kirkland (Procter and Gamble Co., Cincinnati, $\mathrm{OH}, \mathrm{USA})$.

\section{$S D S$-polyacrylamide gel electrophoresis (PAGE)}

The proteins in culture supernates were precipitated with $10 \%$ trichloroacetic acid. The precipitates were collected by centrifugation and resuspended in onefifteenth of the original sample volume of final sample buffer. These samples were electrophoresed by the procedure of Laemmli (1970), modified by the addition of $70 \mathrm{mM} \mathrm{NaCl}$ (final concentration) to the separating gel (Mietzner et al., 1984). The final acrylamide concentration was $12 \%$. Gels were stained with Coomassie blue R250 (Miles Laboratory, Elkhart, IN, USA) $0.125 \%$ in ethanol $40 \%$ and acetic acid $10 \%$. Acetic acid $10 \% \mathrm{v} / \mathrm{v}$ was used to destain the gels.

\section{Western blot analysis}

After electrophoresis, proteins were transferred on to nitrocellulose by the method of Towbin et al. (1979). TSST-1 was detected after incubation with goat antitoxin diluted 1 in 100 . Bound antibody was detected by the method of Blake et al. (1984) with rabbit anti-goat IgG, conjugated to horseradish peroxidase (Miles Laboratories). The developing reagent was 4-chloro-1-naphthol (Sigma).

\section{Amino acid analysis}

Amino acids were determined by the method of Spackman et al. (1958), with a Beckman Model 121 automatic amino acid analyser. Samples were pretreated with trichloroacetic acid (final concentration $10 \%$ ) to precipitate proteins.

\section{Protease assay}

Protease activity was measured with Hide Powder Azure (Calbiochem, La Jolla, CA, USA) as substrate. The reaction mixture consisted of $20 \mathrm{mg}$ of Hide Powder Azure, $400 \mu \mathrm{l}$ of the sample to be tested, and $4.6 \mathrm{ml}$ of $0.05 \mathrm{M}$ tris-hydroxymethyl amino methane buffer, $p \mathrm{H} \mathrm{7.8}$ (Rinderknecht et al., 1968). Tubes were incubated at $37^{\circ} \mathrm{C}$ in a shaking water bath. At 15 -min intervals, samples were removed from the water bath, placed in ice, and passed through a membrane filter. The optical density of each filtrate was determined at $595 \mathrm{~nm}$. 


\section{Miscellaneous determinations}

The total protein was measured in culture supernates, obtained from the chemostat, by the method of Lowry et al. (1951). Egg white lysozyme was used as a standard. Dry weights were determined as previously described (Morse et al., 1974).

\section{Results}

\section{Kinetics of TSST-1 synthesis}

$S$. aureus strains 1169 and 033 were grown in batch culture in CDM, $p \mathrm{H} 6 \cdot 5$. Samples were removed at hourly intervals for the determination of turbidity, dry weight and TSST-1. The results (fig. 1) show that TSST-1 was not detected until the

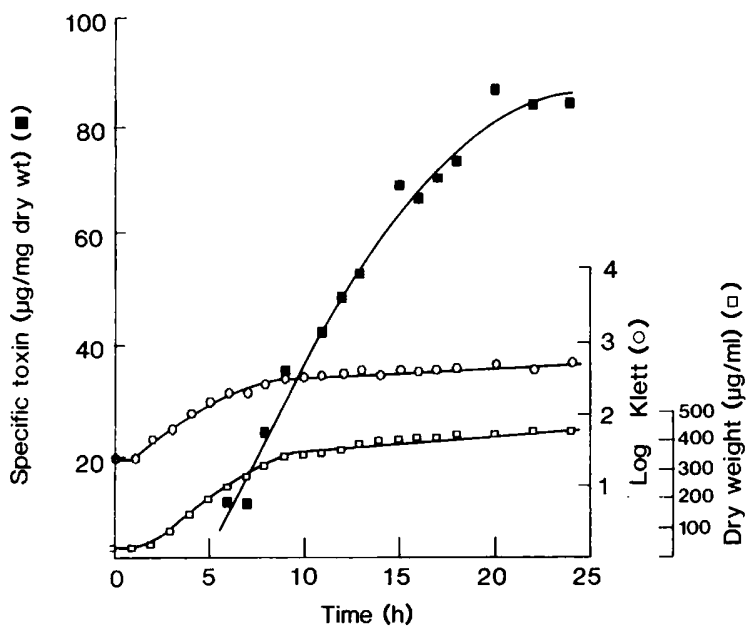

Fig. 1. Kinetics of TSST-1 synthesis by $S$. aureus strain 1169 during batch culture in CDM.

middle of the exponential growth phase; strain 033 exhibited identical kinetics of toxin synthesis (data not shown). TSST-1 was detected after cultures attained a dry weight of about $250 \mu \mathrm{g} / \mathrm{ml}$. Specific toxin rapidly increased as cultures entered the stationary growth phase; it continued to increase during the early stationary phase, at which point cell mass had attained its maximum value. Little additional toxin was synthesised during the late stationary phase.

The differential rate of TSST-1 synthesis by $S$. aureus strain 1169 continued to increase after a critical cell mass was attained (fig. 2). This rapid increase corresponded to the end of the exponential growth phase. The $p \mathrm{H}$ increased from 6.5 to 6.8 during exponential growth.

$S$. aureus strain 1169 synthesised about twice as much TSST-1 as strain 033. Strain 1169 was, therefore, used in subsequent experiments.

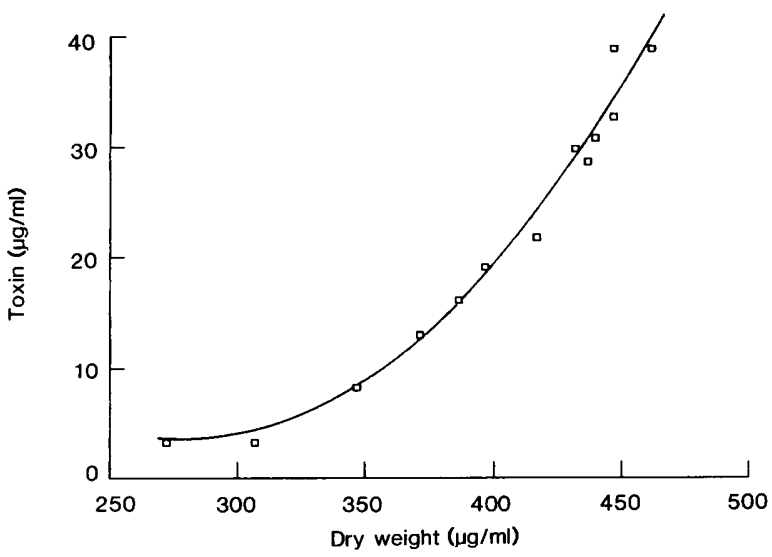

Fig. 2. Differential rate of TSST-1 synthesis during growth of $S$. aureus strain 1169 in CDM.

\section{Effect of aeration on specific TSST-1}

The effect of aeration was examined by growing strain 1169 in volumes of growth medium from 10 to $50 \mathrm{ml}$, in $300-\mathrm{ml}$ nephelometer flasks, with constant agitation at $150 \mathrm{rpm}$. Samples were removed from each flask when growth reached the maximum stationary phase, and TSST- $1, p \mathrm{H}$ and dry weight were measured. The results (fig. 3) indicated that specific toxin increased by c. $200 \%$ with increasing medium volume, that is with decreasing oxygen availability. This was accompanied by a $17 \%$ decrease in the cell yield, and a decrease in the final $p \mathrm{H}$ from $7 \cdot 6$ to $7 \cdot 2$.

\section{Effect of initial pH on TSST-1 synthesis}

Specific toxin was significantly affected by the intitial $p \mathrm{H}$ of the medium (table I). Bacterial growth

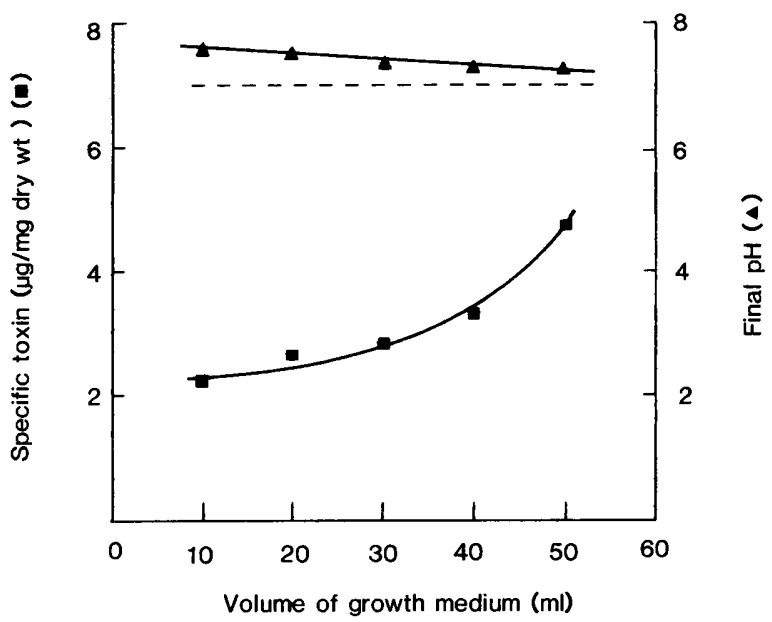

Fig. 3. Effect of aeration on TSST-1 synthesis by $S$. aureus strain 1169 grown in $300 \mathrm{ml}$ nephelometer flasks containing volumes of CDM from 10 to $50 \mathrm{ml}$, with constant agitation at $150 \mathrm{rpm}$. 
Table I. Effect of initial $p \mathrm{H}$ of chemically defined medium on TSST-1 synthesis of $S$. aureus strain 1169

\begin{tabular}{cccc}
\hline Initial $p \mathrm{H}$ & $\begin{array}{c}\text { Final } p \mathrm{H} \\
(17 \mathrm{~h})\end{array}$ & $\begin{array}{c}\text { Corrected cell } \\
\text { yield (mg) }\end{array}$ & $\begin{array}{c}\text { TSST-1 }(\mu \mathrm{g} / \mathrm{mg} \text { dry } \\
\text { weight })\end{array}$ \\
\hline 4.0 & 4.1 & 0 & 0 \\
5.0 & 5.0 & 0 & 0 \\
6.0 & 7.1 & 0.444 & 12.2 \\
6.5 & 7.4 & 0.418 & 18.4 \\
7.0 & 7.6 & 0.460 & 18.5 \\
7.5 & 7.9 & 0.652 & 9.5 \\
8.0 & 8.2 & 0.490 & 10.6 \\
\hline
\end{tabular}

*Dry weight after growth for $17 \mathrm{~h}$ minus dry weight at time 0 .

or toxin synthesis was not observed in medium with an initial $p \mathrm{H} \leq 5 \cdot 0$. The highest cell yield was observed in medium with an initial $p \mathrm{H}$ of 7.5 but the highest specific toxin was observed in medium with an initial $p \mathrm{H}$ between 6.5 and 7.0 . There was no obvious relationship between the final $p \mathrm{H}$ of the medium and the specific toxin.

\section{Effect of anaerobiosis, pyruvate, and magnesium on TSST-1 synthesis in batch culture}

S. aureus strain 1169 grew anaerobically when CDM was supplemented with $40 \mathrm{~mm}$ pyruvate and $0.1 \mathrm{mM}$ uracil. Pyruvate concentration was varied to determine whether it affected TSST-1 synthesis. Fig. 4 shows that the cell yield increased by $c .30 \%$ as the pyruvate concentration increased from 10 to $50 \mathrm{~mm}$ but specific toxin remained relatively unaffected.

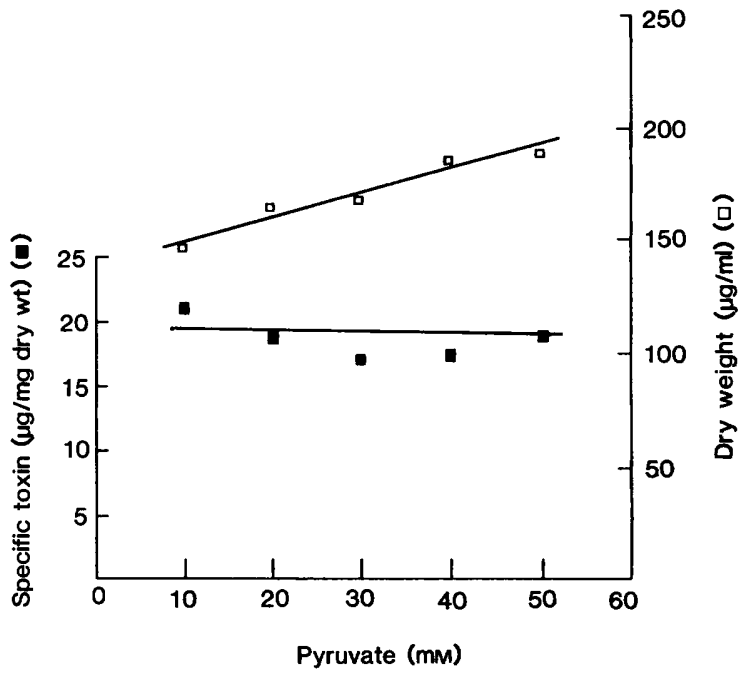

Fig. 4. Effect of pyruvate concentration on TSST-1 synthesis by $S$. aureus strain 1169 grown anaerobically.
Complete CDM contained $0.7 \mathrm{mM} \mathrm{Mg}^{++}$. To study the effect of $\mathrm{Mg}^{++}$on TSST-1 synthesis, the $\mathrm{Mg}^{++}$source was omitted from the medium. Atomic absorption spectroscopy indicated that omitting the $\mathrm{Mg}^{++}$source resulted in a 26-fold reduction in $\mathrm{Mg}^{++}$to $0.027 \mathrm{mM}$. The $\mathrm{Mg}^{++}$ concentration had no effect on the specific toxin in anaerobic conditions (fig. 5) but in aerobic condi-

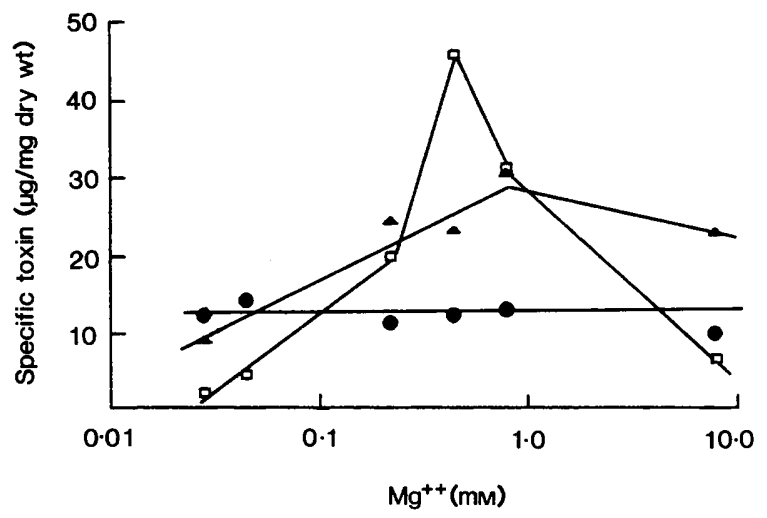

Fig. 5. Effect of $\mathrm{Mg}^{++}$concentration on TSST-1 synthesis by $S$. aureus strain 1169 grown in aerobic and anaerobic conditions: $\square=$ aerobic conditions; $O=$ anaerobic conditions; $\boldsymbol{\Delta}=$ aerobic conditions and CDM supplemented with $40 \mathrm{mM}$ pyruvate and $0 \cdot 1 \mathrm{~mm}$ uracil.

tions, specific toxin increased c. 23-fold with increasing $\mathrm{Mg}^{++}$concentrations up to a concentration of $0.4 \mathrm{mM}$; higher concentrations resulted in a $90 \%$ reduction in specific toxin. In aerobic conditions, the addition of $40 \mathrm{~mm}$ pyruvate and $0.1 \mathrm{~mm}$ uracil to the CDM somewhat modified the effect of $\mathrm{Mg}^{++}$on specific toxin. The specific toxin increased c. 3.5-fold as the $\mathrm{Mg}^{++}$concentration was increased to $0.8 \mathrm{~mm}$; higher concentrations reduced the specific toxin by $25 \%$.

\section{Growth and TSST-1 synthesis in continuous culture}

$S$. aureus strain 1169 was grown in continuous culture in arginine-limiting conditions. A steady state was attained at various dilution rates and the dry weights at each of these dilution rates were determined (table II). Washout occurred at dilution rates greater than $0.45 \mathrm{~h}^{-1}$. The results presented in table II indicate that the highest specific toxin was observed when generation times were increased. During these experiments, the dissolved oxygen concentration remained between 50 and $60 \%$ of saturation. When the dissolved oxygen level was adjusted to $80-100 \%$ of saturation, growth occurred but no TSST-1 was detected (data not shown). This supported previous results obtained 
Table II. Effect of dilution rate on the synthesis of TSST-1, protease and total extracellular protein by S. aureus strain 1169

\begin{tabular}{cccccc}
\hline $\begin{array}{c}\text { Dilution rates } \\
\left(\mathrm{h}^{-1}\right)\end{array}$ & $\begin{array}{c}\text { Dry weight } \\
(\mathrm{mg} / \mathrm{ml})\end{array}$ & $\begin{array}{c}\text { Total extracellular } \\
\text { protein }(\mu \mathrm{g} / \mathrm{ml})\end{array}$ & $\begin{array}{c}\text { Extracellular protein } \\
(\mu \mathrm{g} / \mathrm{mg} \mathrm{dry} \mathrm{weight)}\end{array}$ & $\begin{array}{c}\text { TSST-1 } \\
(\mu \mathrm{g} / \mathrm{mg} \mathrm{dry} \mathrm{weight})\end{array}$ & $\begin{array}{c}\text { Relative protease } \\
\text { activity*/mg dry weight }\end{array}$ \\
\hline 0.05 & $0 \cdot 380$ & 272 & 716 & $4 \cdot 47$ & $24 \cdot 2$ \\
0.08 & 0.355 & 208 & 586 & $2 \cdot 25$ & $240 \cdot 6$ \\
0.11 & 0.295 & 240 & 814 & 2.71 & $338 \cdot 9$ \\
0.15 & 0.160 & 233 & 1456 & 0 & $437 \cdot 5$ \\
\hline
\end{tabular}

${ }^{*}$ Relative protease activity expressed as a percentage of the highest activity observed.

when the effect of aeration on TSST-1 synthesis was examined in batch culture.

SDS-PAGE analysis of samples obtained at the dilution rates given in table II revealed that numerous proteins were present, and there appeared to be both qualitative and quantitative differences between samples obtained at various dilution rates. The gel was electroblotted and the proteins transferred to nitrocellulose as described above. A band corresponding to TSST-1 was apparent after reaction with goat anti-TSST-1, as described previously. Fig. 6 shows that the antiserum reacted specifically with a protein band corresponding to an apparent mol. wt of $c .22000$. The intensity of the bands (fig. 6) supports the data presented in table II. The absence of higher mol. wt species that react with the goat anti-TSST-1 suggests that TSST-1 precursors were not produced in significant amounts in these conditions.

The effect of dilution rate on TSST-1 synthesis was not a general phenomenon applicable to other staphylococcal extracellular proteins. For example, protease activity as a function of dry weight increased with increasing dilution rates (table II).

\section{Discussion}

Several investigators have used continuous culture to study extracellular protein synthesis by $S$. aureus (Engels et al., 1980; Engels and Kamps, 1982). Results have indicated that several extracellular enzymes were produced optimally when $S$. aureus was grown under oxygen-limitation (Jarvis et al., 1973; Carpenter and Silverman, 1974). Our results demonstrated that $S$. aureus strain 1169 , grown in continuous culture with arginine-limitation, produced TSST-1 only when oxygen availability was decreased. A similar relationship between oxygen and TSST-1 synthesis was observed when this strain was grown in batch culture.

The dilution rate has been shown previously to have a varied effect on the synthesis of staphylo-

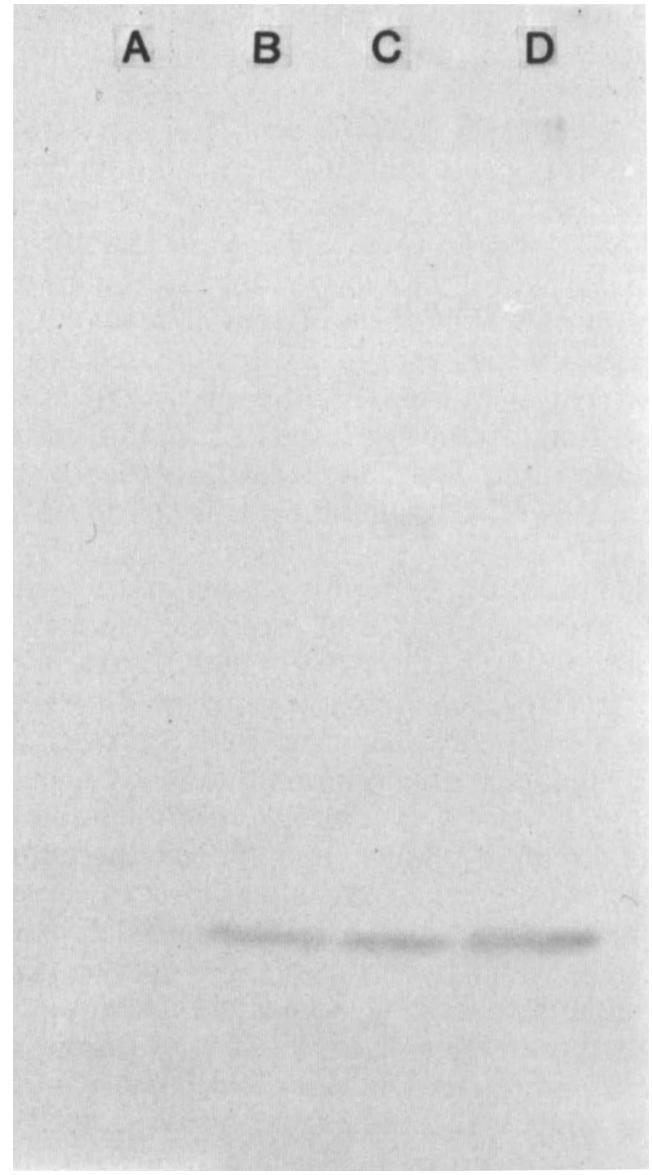

Fig. 6. Western blot of culture supernates of $S$. aureus strain 1169 grown in continuous culture and probed with goat anti-TSST-1. Lanes A-D: dilution rates $0 \cdot 15,0 \cdot 11,0 \cdot 08$ and $0 \cdot 05$, respectively.

coccal extracellular proteins. Jarvis et al. (1975) examined $S$. aureus strain $\mathbf{S 6}$ grown in continuous culture with $\mathrm{Mg}^{++}$limitation and observed that enterotoxin B, lipase, and deoxyribonuclease increased with increasing dilution rates; in contrast, lysozyme decreased. The synthesis of TSST-1 was also affected by the dilution rate. Specific toxin 
decreased as the dilution rate increased; no TSST1 was detected by immunodiffusion at a dilution rate of $\geq 0 \cdot 15 \mathrm{~h}^{-1}$. However, Western-blot reactions with goat anti-TSST-1 indicated that low levels of TSST-1 were produced at a dilution rate of $0 \cdot 15 / \mathrm{h}$. In contrast, the relative protease activity and total extracellular protein, expressed as functions of dry weight, increased 18- and 2-fold, respectively, with increasing dilution rates.

Although the relative protease activity increased substantially with increasing dilution rates, the absolute protease activity remained low (data not shown). This suggested that the decrease in specific toxin observed with increasing dilution rates was not the result of the proteolytic degradation of TSST-1.

The kinetics of TSST-1 synthesis were determined during batch culture. The results supported previous findings (Schlievert, 1985) which indicated that TSST-1 began to accumulate in the medium towards the end of the exponential phase of growth. An examination of the differential rate of toxin synthesis revealed that TSST-1 increased rapidly after a critical cell mass had been attained. This observation, together with the finding that specific toxin increased with decreased availability of oxygen, suggested that the critical cell mass was required to decrease the dissolved oxygen concentration sufficiently to permit optimal toxin synthesis. Other staphylococcal exoproteins such as coagulase (Engels et al., 1980), enterotoxin B, and nuclease (Carpenter and Silverman, 1974) were also produced optimally in oxygen-limited conditions.

The vagina is basically an anaerobic environment (Wagner et al., 1984). The insertion of a tampon was reported to change temporarily the vaginal micro-environment from anaerobic to aerobic (Wagner et al. 1984). The subsequent decrease in oxygen tension may create a suitable microenvironment for TSST-1 synthesis. However, our data demonstrating that TSST-1 synthesis also occurred in anaerobic conditions suggest that factors other than oxygen tension were involved. The specific toxin produced in aerobic conditions in batch cultures was only 4-fold higher than that produced in anaerobic conditions. The results concerning the effect of anaerobiosis on TSST-1 synthesis differed from those obtained by Schlievert and Blomster (1983) who reported a substantial increase in TSST- 1 in aerobic conditions. This discrepancy may have resulted from differences in the media used in these studies. For example, uracil and pyruvate were added to CDM because they are required for the growth of $S$. aureus in anaerobic conditions (Richardson, 1936). Schlievert and
Blomster (1983) used a complex medium containing glucose; they showed that glucose decreased TSST1 synthesis. Furthermore, metabolism of glucose in anaerobic conditions resulted in the production of lactic acid and a concomitant decrease in the $p \mathrm{H}$ (Friedman, 1939).

The initial $p \mathrm{H}$ of the medium markedly affected the synthesis of TSST-1. Maximal specific toxin was observed in medium with an initial $p \mathrm{H}$ between 6.5 and 7.0 ; no growth occurred at $p \mathrm{H} \leq 5.0$. The $p \mathrm{H}$ of the vagina is approximately 4.5 (Rakoff $e t$ $a l ., 1944)$ and increases to about 7 during menstruation (Beller and Schweppe, 1979). Thus, the $p \mathrm{H}$ of the vagina during menstruation is optimal for synthesis of TSST-1.

The effect of $\mathrm{Mg}^{++}$on TSST-1 synthesis has been the subject of several investigations and remains an area of controversy. Mills et al. (1985) reported that the aerobic synthesis of TSST-1 was controlled by the concentration of $\mathrm{Mg}^{++}$, and that the greatest concentration of TSST-1 was observed when the concentration of $\mathrm{Mg}^{++}$was low. Schlievert (1985), was unable to confirm this observation. We have examined the effect of $\mathrm{Mg}^{++}$concentration on specific toxin during aerobic and anaerobic growth in batch culture in CDM. Our results in aerobic conditions are consistent with those of Mills et al. (1985). Even though we determined that the optimum $\mathrm{Mg}^{++}$concentration was two-fold higher than that reported by Mills et al. (1985), a similar effect of $\mathrm{Mg}^{++}$on TSST-1 synthesis was observed. Mills et al. (1985) omitted iron and manganese from their chemically defined medium. Both of these cations were present in CDM and this may account for the differences between our findings and those of Mills et al. (1985). It is possible that iron or manganese or both may also be involved in the regulation of TSST-1 synthesis. However, further studies are needed on the effect of different metals on TSST-1 synthesis. The concentration of $\mathrm{Mg}^{++}$had no effect on the anaerobic synthesis of TSST- 1 in medium supplemented with pyruvate and uracil. To verify that the addition of pyruvate and uracil was not responsible for the differences between the results obtained during growth in aerobic and anaerobic conditions, specific toxin was estimated during aerobic growth in CDM supplemented with pyruvate and uracil. The addition of pyruvate and uracil modified the effect of $\mathrm{Mg}^{++}$on specific toxin. Morse and Baldwin (1973) observed that pyruvate repressed enterotoxin $B$ synthesis in aerobic conditions. The inhibitory effect of pyruvate may depend on the concentration of $\mathrm{Mg}^{++}$.

In a recent communication, Mills et al. (1986) 
addressed the differences between their results and those of Schlievert. Like Mills et al. (1986), we feel that our results are in-vitro observations and that their validity in vivo should be assessed in relation to other environmental factors.

\section{REFERENCES}

Arko R J, Rasheed J K, Broome C V, Chandler F W, Paris A L 1984 A rabbit model of toxic shock syndrome: clinicopathological features. Journal of Infection 8: 205-211.

Beller F K, Schweppe K W 1979 Review on the biology of menstrual blood. In: Beller F K, Schumacher G F B (eds) The biology of the fluids of the female genital tract. Elsevier/ North-Holland, New York, pp 231-245.

Blake M S, Johnston K H, Russell-Jones G J, Gotschlich E C 1984 A rapid, sensitive method for detection of alkaline phosphatase-conjugated anti-antibody on Western blots. Analytical Biochemistry 136: 175-179.

Carpenter D F, Silverman G J 1974 Staphylococcal enterotoxin $B$ and nuclease production under controlled dissolved oxygen conditions. Applied Microbiology 28: 628-637.

Catlin B W 1973 Nutritional profiles of Neisseria gonorrhoeae, Neisseria meningitidis and Neisseria lactamica in chemically defined media and the use of growth requirements for gonococcal typing. Journal of Infectious Diseases 128: 178194.

Crass B A, Bergdoll M S 1986 Toxin involvement in toxic shock syndrome. Journal of Infectious Diseases 153: 918-926.

Engels W, Kamps M A F, Van Boven C P A 1980 Continuous culture studies on the production of staphylocoagulase by Staphylococcus aureus. Antonie van Leeuwenhoek 46: 533550.

Engels W, Kamps M A F 1982 The nature of the competitive ability of spontaneous staphylocoagulase-negative mutants of Staphylococcus aureus with respect to growth of the parent strains in continuous culture. Antonie van Leeuwenhoek 48: 67-83.

Friedemann T E 1939 The carbohydrate metabolism of Staphylococcus aureus. Journal of Biological Chemistry 130: 61-65.

Jarvis A W, Lawrence R C, Pritchard G G 1973 Production of staphylococcal enterotoxins A, B and C under conditions of controlled $\mathrm{pH}$ and aeration. Infection and Immunity 7 : 847-854.

Jarvis A W, Lawrence R C, Pritchard G G 1975 Glucose repression of enterotoxins $A, B$ and $C$ and other extracellular proteins in staphylococci in batch and continuous culture. Journal of General Microbiology 86: 75-87.

Laemmli U K 1970 Cleavage of structural proteins during the assembly of the head of bacteriophage T4. Nature 227: 680685 .

Lowry O H, Rosebrough N J, Farr A L, Randall R J 1951 Protein measurement with the Folin phenol reagent. Journal of Biological Chemistry 193 : 265-275.

Mancini G, Carbonara A O, Heremans J F 1965 Immunochemical quantitation of antigens by single radial immunodiffusion. Immunochemistry 2: 235-254.

Maw J, Meynell G G 1968 The true division and death rates of Salmonella typhimurium in the mouse spleen determined with superinfecting phage P22. British Journal of Experimental Pathology 49 : 597-613.
We thank D. Jue and J. E. McGuffey for the amino acid analyses, E. Sheard for atomic absorption spectroscopy, C. Y. Chen for his expert assistance and M. W. Reeves for helpful suggestions.

Mietzner T A, Luginbuhl G H, Sandstrom E, Morse S A 1984 Identification of an iron-regulated 37,000-dalton protein in the cell envelope of Neisseria gonorrhoeae. Infection and Immunity 45: 410-416.

Mills J T, Parsonnet J, Tsai Y-C, Kendrick M, Hickman R K, Kass E H 1985 Control of production of toxic-shocksyndrome toxin-1 (TSST-1) by magnesium ion. Journal of Infectious Diseases 151: 1158-1161.

Mills J T, Parsonnet J, Kass E H 1986 Production of toxicshock-syndrome toxin-1: effect of magnesium ion. Journal of Infectious Diseases 153: 993-994.

Morse S A, Baldwin J N 1973 Factors affecting the regulation of staphylococcal enterotoxin B. Infection and Immunity 7: 839-846.

Morse S A, Stein S, Hines J 1974 Glucose metabolism in Neisseria gonorrhoeae. Journal of Bacteriology 120 : 702-714.

Polk H C, Miles A A 1973 The decisive period in the primary infection of muscle by Escherichia coli. British Journal of Experimental Pathology 54: 99-109.

Rakoff A E, Feo L G, Goldstein L 1944 The biologic characteristics of the normal vagina. American Journal of Obstetrics and Gynecology $47: 467-494$.

Reeves M W, Pine L, Feeley J C, Wells D E 1984 Presence of toxic shock toxin in toxic shock and other clinical strains of Staphylococcus aureus. Infection and Immunity 46: 590-597.

Richardson G M 1936 The nutrition of Staphylococcus aureus. Necessity for uracil in anaerobic growth. Biochemical Journal 30: 2184-2190.

Rinderknecht H, Geokas M C, Silverman P, Haverback B J 1968 A new ultrasensitive method for the determination of proteolytic activity. Clinica Chimica Acta 21 : 197-203.

Schlievert P M, Blomster D A 1983 Production of staphylococcal pyrogenic exotoxin Type $\mathrm{C}$ : influence of physical and chemical factors. Journal of Infectious Diseases 147: 236242.

Schlievert P M 1985 Effect of magnesium on production of toxicshock-syndrome toxin-1 by Staphylococcus aureus. Journal of Infectious Diseases 152 : 618-620.

Scott D F, Kling J M, Kirkland J J, Best G K 1983 Characterization of Staphylococcus aureus isolates from patients with toxic shock syndrome, using polyethylene infection chambers in rabbits. Infection and Immunity 39: 383-387.

Spackman D H, Stein W H, Moore S 1958 Automatic recording apparatus for use in the chromatography of amino acids. Analytical Chemistry 30: 1190-1206.

Todd J, Fishaut M, Kapral F, Welch T 1978 Toxic-shock syndrome associated with phage-group-I staphylococci. Lancet 2: 1116-1118.

Towbin H, Staehelin T, Gordon J 1979 Electrophoretic transfer of proteins from polyacrylamide gels to nitrocellulose sheets : procedure and some applications. Proceedings of the National Academy of Sciences of the USA 76: 4350-4354.

Wagner G, Bohr L, Wagner P, Petersen L N 1984 Tamponinduced changes in vaginal oxygen and carbon dioxide tensions. American Journal of Obstetrics and Gynecology 148: $147-150$. 